\title{
Spatiotemporal characteristics of the walk-to-run and run-to-walk transition when gradually changing speed
}

\author{
V. Segers ${ }^{\text {a }}$, P. Aerts ${ }^{\mathrm{a}, \mathrm{b}}$, M. Lenoir ${ }^{\mathrm{a}}$, D. De Clercq ${ }^{\mathrm{a}, *}$ \\ ${ }^{a}$ Department of Movement and Sport Sciences, Faculty of Medicine and Health Sciences, \\ University of Ghent, Watersportlaan 2, B-9000 Gent, Belgium \\ ${ }^{\mathrm{b}}$ Laboratory for Functional Morphology, Department of Biology, University of Antwerp, Belgium
}

Received 9 April 2005; received in revised form 12 September 2005; accepted 15 September 2005

\begin{abstract}
The purpose of this study was to examine spatiotemporal parameters of the walk-to-run transition (WRT) and run-to-walk transition (RWT) when speed is altered with different constant accelerations. Twenty women (height: $168.9 \pm 3.36 \mathrm{~cm}$ ) performed three accelerations $\left(0.05,0.07\right.$ and $\left.0.1 \mathrm{~m} \mathrm{~s}^{-2}\right)$ and three decelerations $\left(-0.05,-0.07\right.$ and $\left.-0.1 \mathrm{~m} \mathrm{~s}^{-2}\right)$ on a motor-driven treadmill.

The transition step in the WRT (first step with a flight phase) and RWT (first step with a double stance phase) occurred at the same speed for all accelerations but these did not occur in the same way. The most striking difference was the presence of a transition step with specific spatiotemporal characteristics in the WRT, whereas this was not observed in the RWT.

The transition is not a sudden one-step-event. WRT occurred before transition and consisted of a "pre-transition period" and the transition step whereas RWT occurred after transition and consisted of the transition step and a "post-transition period". Both transition periods were characterized by an exponential evolution of step frequency and step length. Step frequency and step length showed a linear evolution before and after transition.

The flight phase of the transition step in the WRT reached a minimum with comparable duration of the last flight phase in the RWT. The flight phase could be considered as an intrinsic dynamical factor of transition. Further research in kinematics, the trajectory of the body centre of mass and energy fluctuations will give more insight in these transitions.
\end{abstract}

(C) 2005 Elsevier B.V. All rights reserved.

Keywords: Gait transition; Spatiotemporal; Biomechanics

\section{Introduction}

Walking and running differ from each other in the absence or presence of a double stance phase and in the range of speeds [1-3]. Walking has a double stance phase and is more commonly used at lower speeds of locomotion, while running is characterized by a flight phase and is used at higher speeds $[1,4,5]$. When changing speed, humans intuitively change from walking to running or vice versa [6]. Thorstensson and Robertson [6] suggested that this transition is based on previous experience in combination

\footnotetext{
* Corresponding author. Tel.: +32 926463 22; fax: +32 92646484 . E-mail address: Dirk.DeClercq@UGent.be (D. De Clercq).
}

with information from peripheral receptors and the activity in the central networks controlling locomotion.

Recently it has been suggested that locomotion is not strictly controlled by higher executive command structures [7]. According to the dynamical systems approach, locomotion is a pattern emerging from all intrinsic, or physical, properties of the entire locomotion system interacting with the environment and specific task constraints [8-10]. Aerts et al. [11] suggested that this largely self-organised system, or "integrated black box", determines the very specific combination of step frequency and length, i.e. the collective output of the system, at each speed. Changes in this system represent changes of the "integrated black box" or the descending modulation of that black box $[10,11]$. Therefore, when gradually increasing the control 
parameter, e.g. speed, the organisational status of the system is preserved over a wide range of speeds, resulting in the typical walking pattern. However, as speed increases, the order parameter moves away from the walking attractor. This causes the organisational status to become "unstable" which is characterized by an increased movement variability [8,9,12]. At transition, the order parameter changes to the running attractor with a different, but relatively stable, pattern. Therefore, a transition can be seen as a discontinuity in gait [13].

Most researchers believe that transition is an explicit event, based on findings in walking and running at different discrete constant speeds in the proximity of transition [1420]. Li and Hamill [21], however, observed a gradual change in the ground reaction force pattern of the last steps before the transition point in a protocol with gradually changing speed. From that point of view, transition is no longer to be seen as an explicit event but merely as a process. At the transition point, duty factor (ratio of contact time and total stride time) immediately changes but it is not yet known whether or not an adaptation, to complete the transition, follows the transition point. A protocol with gradually changing speed is necessary to determine whether transition is an event or a process. A transition period should be studied to fully comprehend the transition phenomenon. This transition period comprises the transition point - defined as the first step with a flight phase (walk-to-run transition: WRT) or the first step with a double stance (run-to-walk transition: RWT) - together with a number of steps before and after the transition point. In earlier research acceleration was found to be an important task constraint, which influences WRT as well as RWT speed [10]. The amount of acceleration would be one of the factors for hysteresis (WRT speed differs from RWT speed) $[10,18]$. Therefore, different accelerations were incorporated in current study.

The main purpose of this investigation was to describe and interpret spatiotemporal parameters of the walk-to-run and run-to-walk transition period when speed is altered with different constant accelerations. Our hypotheses were: (1) that a transition process is visible in the spatiotemporal characteristics of several steps before and after the transition point and (2) that the WRT is different from the RWT.

\section{Materials and method}

\subsection{Subjects}

A group of 20 active, normal female human subjects participated in the study having given informed consent. Average values and standard deviations for age, height and mass can be found in Table 1. Subjects were selected on sex, height, being minimal $1.65 \mathrm{~m}$ and maximal $1.75 \mathrm{~m}$ to rule out any possible influence of height and leg length, although only weak correlations have been found between anthropometric variables and transition speed [6,15,17-19]. The
Table 1

Subjects characteristics: mean $(X)$ and standard deviation (S.D.) for height, body mass, leg length and age

\begin{tabular}{lrr}
\hline & \multicolumn{1}{l}{ X } & S.D. \\
\hline Height $(\mathrm{cm})$ & 168.9 & 3.36 \\
Body mass $(\mathrm{kg})$ & 63.2 & 5.98 \\
Leg length $(\mathrm{cm})^{\mathrm{a}}$ & 91.4 & 1.80 \\
Age (years) & 24.5 & 2.76 \\
\hline
\end{tabular}

${ }^{\mathrm{a}}$ Leg length $=$ distance trochanter major-ground.

ethical committee of the Ghent University Hospital approved the experimental protocol.

\subsection{Treadmill protocol}

Before the tests all subjects were familiarised with the treadmill by using it for at least $15 \mathrm{~min}$ at different speeds [22].

Each subject performed 25 trials divided into five blocks of five trials with a rest period of $30 \mathrm{~s}$ between each block, after one familiarisation trial block. Each block was characterized by a specific constant acceleration and were $1 \mathrm{P}$ $\left(a=0.1 \mathrm{~m} \mathrm{~s}^{-2}\right), 5 \mathrm{P}\left(a=0.05 \mathrm{~m} \mathrm{~s}^{-2}\right), 7 \mathrm{P}\left(a=0.07 \mathrm{~m} \mathrm{~s}^{-2}\right)$, $1 \mathrm{~N} \quad\left(a=-0.1 \mathrm{~m} \mathrm{~s}^{-2}\right), \quad 5 \mathrm{~N} \quad\left(a=-0.05 \mathrm{~m} \mathrm{~s}^{-2}\right)$ and $7 \mathrm{~N}$ $\left(a=-0.07 \mathrm{~m} \mathrm{~s}^{-2}\right)$. 'P' and ' $\mathrm{N}$ ' indicate positive and negative acceleration, respectively, causing walk-to-run transitions (WRT) and run-to-walk transitions (RWT). By choosing these magnitudes, the acceleration at which the WRT speed equals probably the RWT speed, i.e. no hysteresis at $0.07 \mathrm{~m} \mathrm{~s}^{-2}$ [10] is included as well as lower $\left(0.05 \mathrm{~m} \mathrm{~s}^{-2}\right)$ and higher $\left(0.1 \mathrm{~m} \mathrm{~s}^{-2}\right)$ values. The blocks were divided at random over the subjects but alternating a $\mathrm{P}$ with an N-block. The first block was considered a familiarisation trial block and was not incorporated in the calculations.

The speed of the treadmill was electronically registered $(5 \mathrm{~Hz})$ on-line and synchronized with video recordings by means of LEDs.

\subsection{Video recordings}

Sagittal plane films using a high-speed video camera (JVC DVL9800) at 200 frames/s were taken of all trials and focussed on the leg movements. The moment of initial contact and of final contact of the foot with the treadmill were determined from the video recordings [23] (maximal error $=0.01 \mathrm{~s}$ ). This permitted the analysis of a step, the smallest functional physiological increment that represents changes in spatiotemporal output. The following spatiotemporal parameters were calculated [24]:

Duty factor $(\mathrm{df})=$ ratio of contact time and total stride time (period between two heel strikes of the same foot). Step frequency $(\mathrm{sf})=$ number of steps over a period of time, calculated as $1 / \Delta t(\Delta t$ : time between two successive foot contacts). 
Step length $(\mathrm{sl})=$ distance travelled from heel strike of one foot to the heel strike of the other foot (treadmill speed divided by step frequency).

Double stance phase $=$ period in a walking stride with both feet touching the ground.

Distance of double stance phase $=$ double stance duration multiplied with the instantaneous speed of the treadmill. Flight phase $=$ period in a running stride with both feet in the air.

Distance of flight phase $=$ flight phase duration multiplied with the instantaneous speed of the treadmill.

\subsection{Statistics}

All data were analysed using the SPSS 11.0 package. Descriptive statistics (mean \pm S.D.) were calculated for subject characteristics, speed $(v)$, duty factor (df), step frequency (sf) and step length (sl). The analyses to compare $v$, df, sf and sl were done in a step-by-step manner. The transition step was named step zero (0) and defined as the first step with a flight phase when speed was increased (WRT) or the first step with a double stance phase when speed was decreased (RWT). Before transition, steps were given negative signs; steps after transition were given positive signs. For each condition the average of all successfully recorded trials (minimum three, maximum five) was used since intra-subject variability was low (see Section 3). Therefore, intra-class correlation coefficients (ICCs) were calculated separately for each individual and for every acceleration/deceleration. In the transition period (step -8 until step +8$)$ a best fit through least squares regression (linear and polynome of second order) was calculated.

A two (negative versus positive acceleration) by three (high $0.1 \mathrm{~m} \mathrm{~s}^{-2}$, intermediate $0.07 \mathrm{~m} \mathrm{~s}^{-2}$, low $0.05 \mathrm{~m} \mathrm{~s}^{-2}$ acceleration) repeated measures analysis of variance (ANOVAs) was used to test the effects of sign and magnitude of the acceleration. A paired samples $T$-test was then used to examine the differences in duty factor, step frequency and step length between steps -1 and 0 and between steps 0 and +1 . Slopes were calculated for every individual at each level. A two (before and after transition) $\times$ three (acceleration) repeated measures ANOVA was used to examine possible differences before and after transition and between accelerations in the WRT and in the RWT.

\section{Results}

\subsection{Intra-variability}

The intra-variability was very low for speed, duty factor, step frequency and step length which was indicated by the high ICCs which were never lower than 0.93 for all subjects. Because of the high ICC, the average of each subject could
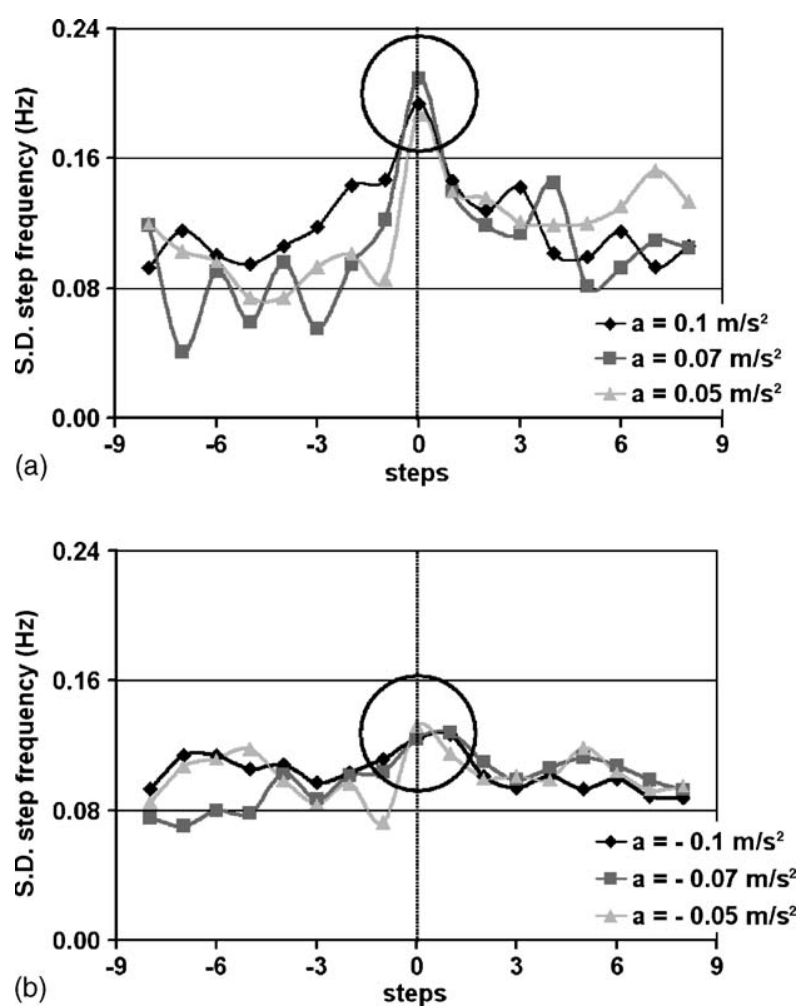

Fig. 1. Intra-subject variability. The evolution of the intra-subject variability by means of the standard deviation on step frequency in the (a) WRT and (b) RWT.

be used instead of the separate trials. Intra-subject variability for step frequency is indicated in Fig. 1.

\subsection{Transition speed}

There was no significant difference for transition speed between the six different conditions (Table 2). Transition was not affected by the sign of acceleration $\left(F_{1,15}=1.744\right.$; $p=0.206)$ nor by the magnitude of acceleration $\left(F_{2,30}\right.$ $=1.981 ; p=.175)$. The repeated measures ANOVA did not reveal any interaction effect either $\left(F_{2,30}=.185 ; p=.832\right)$.

\subsection{Duty factor}

In the protocols with increasing speed, duty factor slightly decreased from step -8 until step -1 before

Table 2

Transition speed: mean $(X)$ and standard deviation (S.D.)

\begin{tabular}{llll}
\hline & \multicolumn{2}{l}{ Transition speed $\left(\mathrm{m} \mathrm{s}^{-1}\right)$} & \\
\cline { 2 - 4 } & $a\left(\mathrm{~m} \mathrm{~s}^{-2}\right)$ & $X$ & S.D. \\
\hline \multirow{2}{*}{ WRT } & 0.1 & 2.16 & 0.12 \\
& 0.07 & 2.10 & 0.06 \\
& 0.05 & 2.12 & 0.08 \\
RWT & -0.1 & 2.19 & 0.14 \\
& -0.07 & 2.12 & 0.09 \\
& -0.05 & 2.17 & 0.06 \\
\hline
\end{tabular}



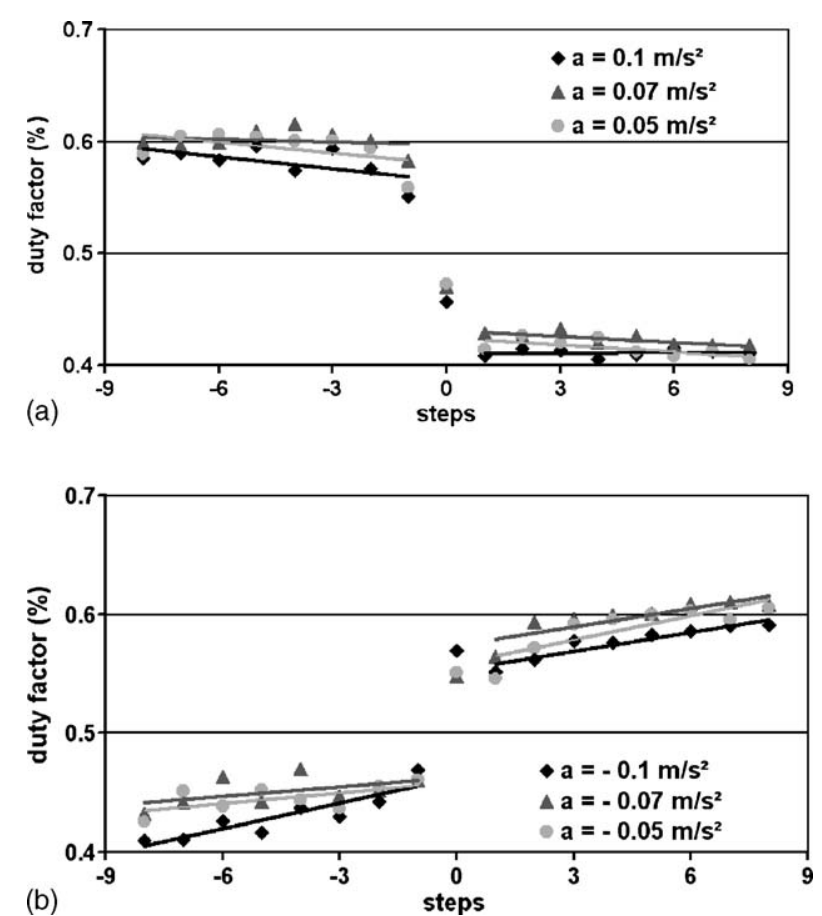

Fig. 2. Duty factor. The evolution of mean duty factor in the (a) WRT and (b) RWT is represented.

transition in a linear fashion (Fig. 2a). In step -1 , the duty factor then fell from approximately 0.58 to the significantly lower value 0.46 in the transition step (Table $3 a$ ), to decrease further significantly to 0.42 in step +1 after transition. After transition (step +1 until step +8 ) the duty factor slightly decreased (Fig. 2a). Slopes remained the same for all accelerations, but a significant difference was found between the slope before and after transition.

The opposite was observed in the protocols with decreasing speed. Before transition, duty factor increased slightly, then suddenly increased significantly (Table 3a) from approximately 0.46 in step -1 to 0.55 in step +1 (Fig. 2b). There was no difference between the slopes before and after transition or between accelerations.

\subsection{Step frequency-step length}

In the WRT-protocol, the evolution of step frequency and step length in the last walking steps (Fig. 3) was best fitted with second order polynomes. The last two walking steps were characterized by an increased step frequency and decreased step length. Step frequency and step length in the transition step were significantly different from both the last walking and the first running step (Table $3 \mathrm{~b}$ and c) showing a clear discontinuity in the collective output of the system. After transition, the spatiotemporal characteristics of the first running steps evolved in an opposite but linear way. A slightly decreasing step frequency and increasing step length was observed.

In the RWT-protocol (Fig. 4) step frequency and step length of the last running steps before transition had a linear evolution, whereas a second order polynome best described the evolution during the first walking steps after transition.

Table 3

Average $(X)$ and standard deviation (S.D.) for steps $-1,0$ and +1

\begin{tabular}{|c|c|c|c|c|c|c|c|c|c|c|c|c|c|}
\hline & \multirow[t]{2}{*}{$a\left(\mathrm{~m} \mathrm{~s}^{-2}\right)$} & \multicolumn{5}{|c|}{ Step -1} & \multicolumn{2}{|c|}{ Step 0} & \multicolumn{5}{|c|}{ Step +1} \\
\hline & & $\bar{X}$ & S.D. & $T^{\mathrm{a}}$ & $\mathrm{df}^{\mathrm{a}}$ & $p^{\mathrm{a}}$ & $\bar{X}$ & S.D. & $\bar{X}$ & S.D. & $T^{\mathrm{b}}$ & $\mathrm{df}^{\mathrm{b}}$ & $p^{\mathrm{b}}$ \\
\hline \multicolumn{14}{|c|}{ (a) Results of paired sample $T$-test between steps $0,-1$ and +1 for duty factor } \\
\hline \multirow[t]{3}{*}{ WRT } & 0.1 & 0.55 & 0.023 & 12.838 & 15 & $<0.01$ & 0.46 & 0.034 & 0.41 & 0.034 & 5.062 & 15 & $<0.01$ \\
\hline & 0.07 & 0.58 & 0.013 & 17.309 & 15 & $<0.01$ & 0.47 & 0.015 & 0.43 & 0.024 & 7.141 & 15 & $<0.01$ \\
\hline & 0.05 & 0.56 & 0.033 & 10.335 & 15 & $<0.01$ & 0.47 & 0.028 & 0.41 & 0.024 & 6.27 & 15 & $<0.01$ \\
\hline \multirow[t]{3}{*}{ RWT } & -0.1 & 0.47 & 0.029 & -13.181 & 16 & $<0.01$ & 0.57 & 0.026 & 0.55 & 0.019 & 4.211 & 16 & $<0.01$ \\
\hline & -0.07 & 0.46 & 0.016 & -8.146 & 16 & $<0.01$ & 0.55 & 0.030 & 0.56 & 0.016 & -1.306 & 16 & $\mathrm{~ns}$ \\
\hline & -0.05 & 0.46 & 0.017 & 1.57 & 16 & $\mathrm{~ns}$ & 0.55 & 0.019 & 0.55 & 0.020 & 1.185 & 16 & $\mathrm{~ns}$ \\
\hline \multicolumn{14}{|c|}{ (b) Results of paired sample $T$-test between steps $0,-1$ and +1 for step frequency } \\
\hline \multirow[t]{3}{*}{ WRT } & 0.1 & 1.96 & 0.22 & -10.15 & 15 & $<0.01$ & 2.37 & 0.29 & 2.04 & 0.22 & 6.32 & 15 & $<0.01$ \\
\hline & 0.07 & 1.90 & 0.18 & -14.17 & 15 & $<0.01$ & 2.41 & 0.31 & 1.97 & 0.21 & 11.99 & 15 & $<0.01$ \\
\hline & 0.05 & 1.87 & 0.13 & -12.29 & 15 & $<0.01$ & 2.41 & 0.28 & 2.02 & 0.21 & 6.60 & 15 & $<0.01$ \\
\hline \multirow[t]{3}{*}{ RWT } & -0.1 & 1.98 & 0.17 & -2.13 & 16 & $\mathrm{~ns}$ & 2.03 & 0.19 & 1.98 & 0.19 & 1.39 & 16 & $\mathrm{~ns}$ \\
\hline & -0.07 & 1.97 & 0.16 & -0.11 & 16 & ns & 1.97 & 0.19 & 2.03 & 0.19 & -2.08 & 16 & $\mathrm{~ns}$ \\
\hline & -0.05 & 1.98 & 0.10 & 1.57 & 16 & ns & 1.96 & 0.20 & 2.06 & 0.17 & -5.94 & 16 & $<0.01$ \\
\hline \multicolumn{14}{|c|}{ (c) Results of paired sample $T$-test between steps $0,-1$ and +1 for step length } \\
\hline \multirow[t]{3}{*}{ WRT } & 0.1 & 1.10 & 0.05 & 9.023 & 15 & $<0.01$ & 0.91 & 0.07 & 1.09 & 0.03 & -6.94 & 15 & $<0.01$ \\
\hline & 0.07 & 1.09 & 0.07 & 7.194 & 15 & $<0.01$ & 0.87 & 0.12 & 1.04 & 0.07 & -8.367 & 15 & $<0.01$ \\
\hline & 0.05 & 1.12 & 0.06 & 12.373 & 15 & $<0.01$ & 1.12 & 0.06 & 1.07 & 0.08 & -7.772 & 15 & $<0.01$ \\
\hline \multirow[t]{3}{*}{ RWT } & -0.1 & 1.12 & 0.07 & 3.532 & 16 & $<0.01$ & 1.08 & 0.09 & 1.09 & 0.09 & -0.598 & 16 & $\mathrm{~ns}$ \\
\hline & -0.07 & 1.08 & 0.08 & -0.406 & 16 & $\mathrm{~ns}$ & 1.09 & 0.05 & 1.02 & 0.07 & 6.58 & 16 & $<0.01$ \\
\hline & -0.05 & 1.11 & 0.06 & 0.358 & 16 & $\mathrm{~ns}$ & 1.11 & 0.05 & 1.04 & 0.06 & 7.569 & 16 & $<0.01$ \\
\hline
\end{tabular}

${ }^{\text {a }}$ Comparison between transition step and step -1 .

b Comparison between transition step and step +1 . 

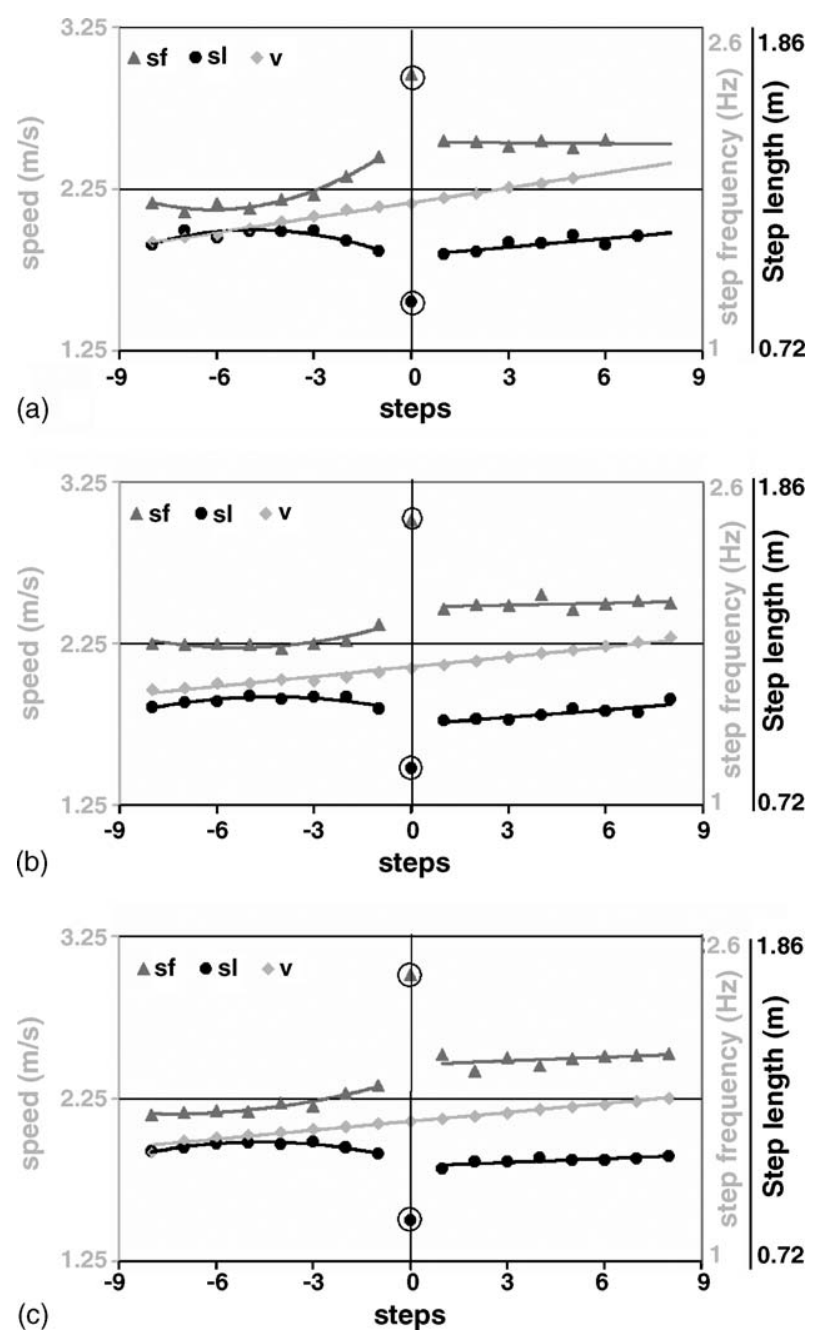

Fig. 3. Spatiotemporal factors for the walk-to-run transition (WRT). The evolution of mean step frequency (sf), mean step length (sl) and mean speed can be seen for the different accelerations (a) $0.1 \mathrm{~m} \mathrm{~s}^{-2}$, (b) $0.07 \mathrm{~m} \mathrm{~s}^{-2}$ and (c) $0.05 \mathrm{~m} \mathrm{~s}^{-2}$. Regression lines have $R^{2}$ values ranging between 0.27 and 0.86 .

Before transition there was a decrease in both step frequency and step length. The transition step was closely related to the last running step (step -1 ), as can be seen in Table 3. In comparison to the first walking step $($ step +1$)$ the step frequency was altered. After transition there was a substantial decrease in step frequency and increase in step length. This latter increase reached a peak at step +4 , with step frequency decreasing slightly and step length remaining relatively constant thereafter.

\subsection{Flight phase and double stance}

In WRT-protocol the last walking steps before transition had a reduced double stance. In the RWT-protocol flight phase also decreased before transition.

There was no significant difference between the flight phase duration of the last step before transition in the RWTprotocol (Fig. 5b) and the first step after transition in the
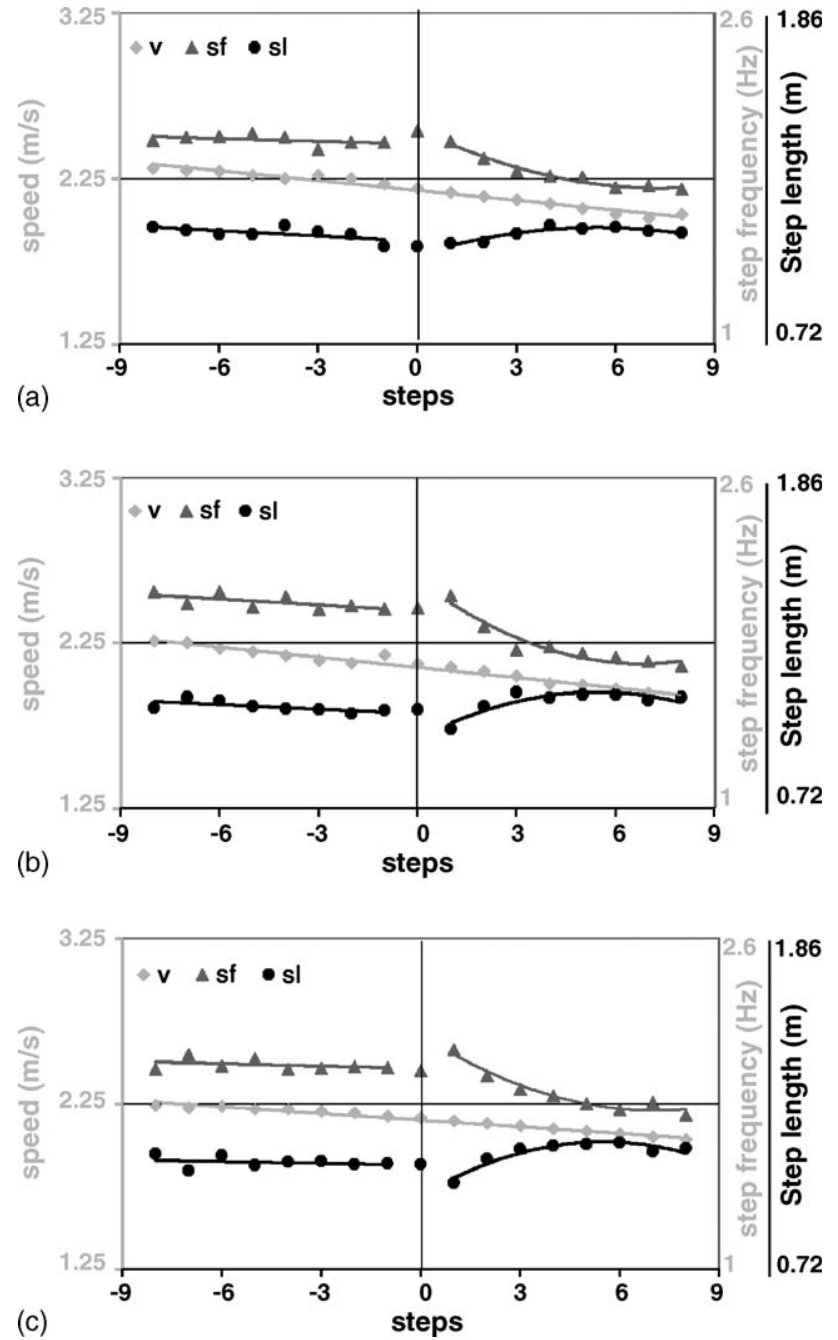

Fig. 4. Spatiotemporal factors for the run-to-walk transition (RWT). The evolution of mean step frequency (sf), mean step length (sl) and mean speed can be seen for the different accelerations (a) $-0.1 \mathrm{~m} \mathrm{~s}^{-2}$, (b) $-0.07 \mathrm{~m} \mathrm{~s}^{-2}$ and (c) $-0.05 \mathrm{~m} \mathrm{~s}^{-2}$. Regression lines have $R^{2}$ values ranging between 0.26 and 0.97 .

WRT-protocol (Fig. 5a). On the other hand the double stance of the last step before transition in the WRT-protocol (Fig. 5a) was significantly longer than the first double stance in RWTprotocol (Fig. 5b) in two of the three accelerations $(p<0.01)$. In the WRT-protocol the flight phase of the transition step was significantly shorter than the double stance of step -1 and the flight phase of step +1 . In the RWT-protocol the double stance of the transition step was shorter than the flight phase of step -1 , before transition $(p<0.01)$ (Fig. 5b).

After transition there was a gradual increase in the duration of flight phase in WRT and of double stance in the RWT.

\section{Discussion}

A transition process was present in all accelerations and differences were observed between the WRT and the RWT 

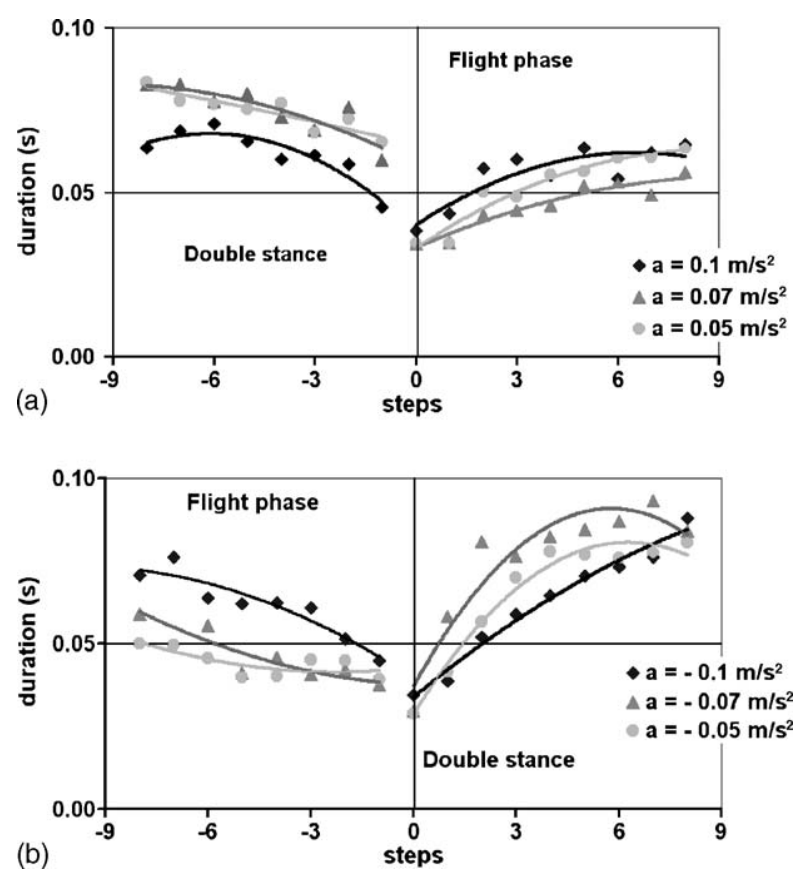

Fig. 5. Duration of double stance phase and flight phase. Duration of flight phase and stance phase in the (a) WRT and (b) RWT for the three different accelerations/decelerations. $R^{2}$ values for the regression line for flight phase vary between 0.89 and 0.97 in the WRT and between 0.05 and 0.16 in the RWT. $R^{2}$ values are low but the regression lines only have an illustrative value. An exponential relationship is chosen because linear regression had even smaller $R^{2}$ values. $R^{2}$ values for the regression line for double stance phase vary between 0.59 and 0.88 in the WRT and between 0.76 and 0.94 in the RWT.

indicating that our hypotheses were confirmed. This transition process seemed very stable since both intrasubject and inter-subject variability were low. Inter-subject variability was not reported separately but can be seen for the interval steps -1 to +1 in Table 3 in Fig. 6 .

\subsection{Walk-to-run transition (WRT)}

An exponential relationship in step frequency and step length was found from step -8 to the transition step (Fig. 3), in contradiction to the linear evolution described previously [4,24-26]. To consider this further, we examined step frequency and step length in the interval steps -15 to -8 , where a linear evolution of step frequency and step length was found (Fig. 6a). Due to technical limitations, recordings were limited to 8 seconds and data of the interval steps -15 to -8 were only available for six subjects.

WRT was not a sudden event but more of a process consisting of a "pre-transition period" and the transition step. The pre-transition period was situated from steps -8 to -1 , since the linear evolution of step frequency and step length changed at that point into an exponential evolution. The $R^{2}$ values of the exponential regression were highest starting at step -8 . Of importance is that a transition process exists, rather than knowing its exact starting point.
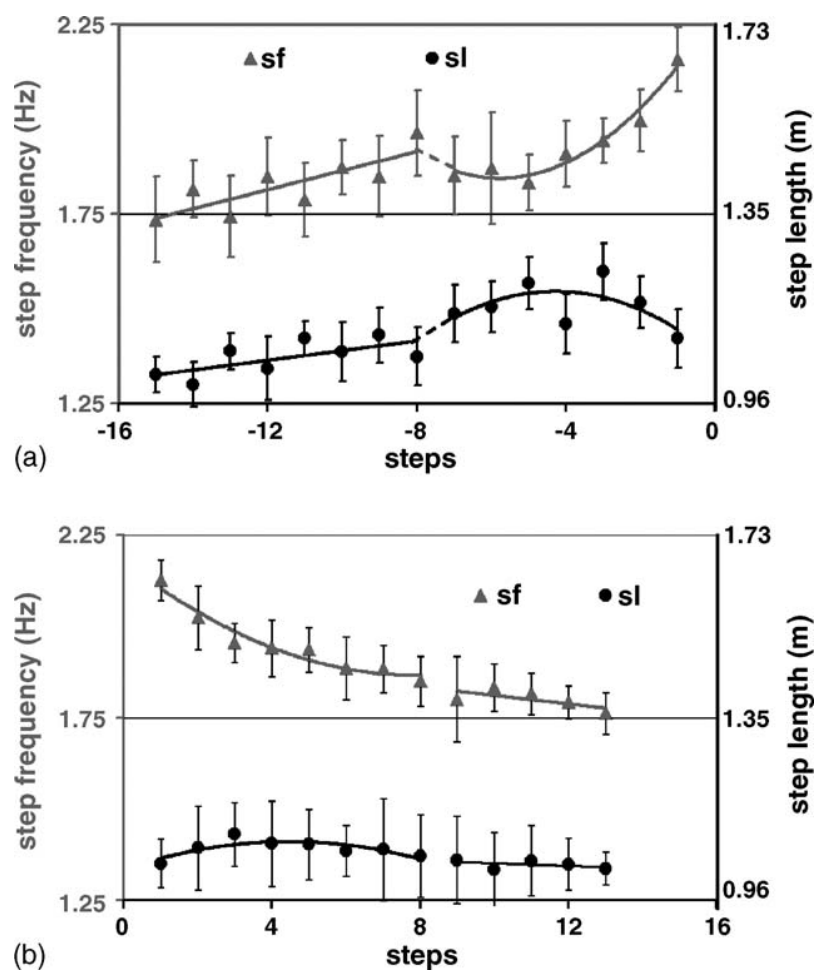

Fig. 6. Evolution of step length and step frequency. The evolution of step length and step frequency (with standard deviation indicated) for (a) six subjects from 15 steps before transition in the WRT-protocol. $R^{2}$ for the lines of regression vary between 0.57 and 0.94 . (b) Five subjects from transition in the RWT-protocol to 15 steps after transition with $R^{2}$ varying between 0.62 and 0.87 .

The most striking event in the WRT was the outlying transition step (Figs. 2 and 3, Table 3). Since it is the first step with a flight phase, the duty factor dropped below 0.5 , and this step is different from the following running step. Moreover, step 0 was an outlier for step frequency and step length. Due to these specific spatiotemporal characteristics the transition step could neither be classified under walking nor under running and probably is a key factor in the conversion from walking to running. Because of the presence of a flight phase, this step was defined kinematically as a running step but this assumption should be regarded with caution as this step's spatiotemporal behaviour (duty factor, step frequency and step length) was significantly different from step +1 (Table 3 ).

After the transition point, when running, a linear evolution of step frequency and step length was observed, (Fig. 3) as expected in submaximal running [27]. Increase in speed was mainly due to a larger step length $[27,28]$, and was accomplished mostly by the increasing distance covered during the flight phase.

Using the dynamical systems theory, it could be concluded that the walking pattern is drifting away from the walking attractor throughout the last steps before the transition step, where the control parameter, e.g. speed, reaches its critical value [8-10,20,21]. The locomotion 
system moved through an unstable region, situated approximately between steps -8 and 0 . The coordination pattern abruptly changed at the transition point to the running attractor $[8,9]$. At critical values of the control parameter, the order parameter (step frequency) underwent a major change in value and was accompanied by an increased variability, as has been noted previously $[8,9,20]$. These socalled critical fluctuations are visible in Fig. 1a. The transition resulted in a rise in step frequency, a drop in step length and an increase in variability in line with the predictions of the attractor theory [8].

\subsection{Run-to-walk transition (RWT)}

The last steps before the transition step were characterized by a decrease in step length and a less pronounced decrease in step frequency. This linear evolution of step frequency and step length (Fig. 4) is in line with earlier findings of spatiotemporal characteristics of submaximal running [27]. The transition step follows the evolution of step length and step frequency of the last running steps. Because of the presence of a first double stance, with a comparable duration of the last flight phase, the duty factor immediately rose above 0.5 (Table 3 ). As the system moved to the transition point the typical critical fluctuations were not observed, in contrast to the findings in the WRT (Fig. 1b).

After the transition point, step frequency increased in the first walking step, then decreased exponentially in a period of 6-7 steps, and vice a versa for step length (Fig. 4). For the same reason as in the WRT, the period following the transition was examined (steps +8 to +15 ). This additional information was only obtained in five subjects because of technical limitations. Depending on the individual, the linear evolution of step frequency and step length started at steps +6 or +7 (minimum root mean square). The exact timing of the process is less important than the recognition of the existence of the RWT process, which consists of the transition step and a "post-transition period".

\subsection{Transition step(s): functional hysteresis}

In the present research, WRT and RWT speeds did not differ and different accelerations in both transitions did not lead to other transition speeds. This was in contrast with the findings of $\mathrm{Li}$ [10] who identified acceleration as an important task constraint determining transition speed. The difference might be explained by the fact that acceleration is only one among many constraints, such as the chosen population. In the current study a homogeneous population of trained women was chosen to eliminate any bias that might be seen in the heterogeneous population studied by $\mathrm{Li}$ [10].

In the present study no hysteresis in the strict sense of its definition was found as transition speed in the WRT and RWT protocols did not differ. However, a "functional hysteresis" was observed: WRT and RWT are realized another way. Firstly, we have shown that a transition step was present in WRT and not RWT. In line with the findings of Lee and Farley [29], the transition step in the WRT might enable the locomotion system to accomplish the greater compression of the standing leg (more knee flexion). Secondly, transition from one mode of locomotion to another took place in the walking steps close to transition as well in the WRT (before the transition point) as in the RWT (after the transition point). The spatiotemporal nature of the running pattern was more likely to be related to the unique step frequency-step length combination at each speed, even in proximity of the transition point, which could be interpreted as the strength of the running attractor. The term 'functional hysteresis' may be illustrated by considering comparisons between 'equivalent' steps. The first step with a flight phase in the WRT-protocol (step 0) was compared to step -1 in the RWT protocol and so on. The running steps did not differ. The walking steps in the transition period, on the other hand, showed significant differences, indicating that the adaptation to running (WRT) differed from the adaptation to walking (RWT).

\subsection{Trigger}

One intriguing question in gait transitions is to consider what triggers an alteration in a locomotion pattern? Hreljac [16] formulated four criteria in order to label a variable as trigger. The variable had to (1) change abruptly to a (2) different value at a (3) critical point that had to remain (4) constant in different conditions.

The flight phase reached a minimum at the transition point in both the WRT and RWT protocols. The last flight phase in the WRT-protocol was not significantly different from the first flight phase in RWT-protocol. The transition step was launched as soon as the minimal duration of flight could be generated in the WRT. Double stance appeared whenever the flight phase duration could not decrease any further in the RWT. The flight phase can be considered an intrinsic dynamical constraint of human locomotion [30,31]. It is likely that the integrated black box [11] was then stimulated to undergo a modulation based on the intrinsic dynamical characteristic.

WRT and RWT do not occur at the same point in time and are more likely to be a process, as is the case in some animals $[32,33]$. The steps in the transition process have a double stance and an exponential evolution of step frequency and step length. A possible explanation could be that the system output adapts to produce the most efficient transition possible. However, it is not possible to explain fully the exact mechanism based on spatiotemporal factors alone. Further research in kinematics, the trajectory of the centreof-mass and energy fluctuations in this transition zone might help a better understanding of the transition phenomenon.

In conclusion, the WRT and RWT processes were not the same. Adaptation to changing task constraints takes place 
primarily in the walking steps close to the transition both in WRT and in RWT and results in a pre- and post-transition period, respectively. In WRT an outlying transition step was observed, whereas no such step was seen in the RWT. The flight phase reached a minimum at the transition point and could be considered an intrinsic dynamical factor.

\section{Acknowledgements}

This research was supported by BOF-RUG B/03796/01IV1. The authors acknowledge Ing. P. Van Cleven and Ing. D. Spiessens for data collection and technical support and Lic. A. De Cock for her professional advice. The authors also thank Prof. Dr. D. Adriaens (Ghent University, department of Vertebrate Morphology and Zoology Museum) for the use of the JVC high-speed camera.

\section{References}

[1] Getchell N, Whitall J. Transitions to and from asymmetrical gait patterns. J Motor Behav 2004;36:13-27.

[2] Whitall J, Caldwell GE. Coordination of symmetrical and asymmetrical human gait. J Motor Behav 1992;24:339-53.

[3] Titianova EB, Mateev PS, Tarkka IM. Footprint analysis of gait using a pressure sensor system. J Electromyogr Kinesiol 2004;14:275-81.

[4] Winter DA. The biomechanics and motor control of human gait: normal, elderly and pathological, 2nd ed., University of Waterloo Press; 1991.

[5] Farley CT, Ferris DP. Biomechanics of walking and running: center of mass movements to muscle action. Exercise Sport Sci Rev 1998; 26:253-68.

[6] Thorstensson A, Roberthson H. Adaptations to changing speed in locomotion: speed of transition between walking and running. Acta Physiol Scand 1987;131:211-4.

[7] Davids K, Handford C, Williams M. The natural physical alternative to cognitive theories of motor behavior: an invitation for interdisciplinary research in sport science? J Sport Sci 1994;12:495-528.

[8] Diederich FJ, Warren WH. Why change gaits? Dynamics of the walkrun transition. J Exp Psychol Human 1995;21:183-202.

[9] Diederich FJ, Warren WH. Dynamics of gait transitions: effects of grade and load. J Motor Behav 1998;30:60-78.

[10] Li L. Stability landscapes of walking and running near gait transition speed. J Appl Biomater 2000;16:428-35.

[11] Aerts P, Van Damme R, Van Elsacker L, Duchene V. Spatio-temporal gait characteristics of the hind-limb cycles during voluntary bipedal and quadrupedal walking in bonobos (Pan paniscus). Am J Phys Anthropol 2000;111:503-17.

[12] van Emmerik REA, van Wegen EEH. On variability and stability in human movement. J Appl Biomater 2000;16:394-406.
[13] Alexander RMcN. Optimization and gaits of locomotion in vertebrates. Physiol Rev 1989;4:1199-227.

[14] Nilsson J, Thorstensson A. Adaptability in frequency and amplitude of leg movements during human locomotion at different speeds. Acta Physiol Scand 1987;129:107-14.

[15] Hreljac A. Effects of physical characteristics on the gait transition speed during human locomotion. Hum Movement Sci 1995;14: 205-16.

[16] Hreljac A. Determinants of the gait transition speed during human locomotion: kinematic factors. J Biomech 1995;28:669-77.

[17] Getchell N, Whitall J. Transitions in gait as a function of physical parameters. J Sport Exercise Psychol 1997;19:S55.

[18] Hanna A, Abernethy B, Neal RJ, Burgess-Limerick R. Triggers for the transition between human walking and running. In: Sparrow WA, editor. Energetics of human activity. Champaign, IL: Human Kinetics; 2000. p. 124-164.

[19] Abernethy B, Hanna A, Plooy A. The attentional demands of preferred and non-preferred gait patterns. Gait Posture 2002;15:250-65.

[20] Raynor AJ, Yi CJ, Abernethy B, Jong QJ. Are transitions in human gait determined by mechanical, kinetic or energetic factors? Hum Movement Sci 2002;21:785-805.

[21] Li L, Hamill J. Characteristics of the vertical ground reaction force component before gait transition. Res Q Exercise Sport 2002;73: 229-37.

[22] Wall JC, Charteris J. The process of habituation to treadmill walking at different velocities. Ergonomics 1980;23:425-35.

[23] Wall JC, Crosbie J. Accuracy and reliability of temporal gait measurement. Gait Posture 1996;4:293-6.

[24] Zatsiorsky VM, Werner SL, Kaimin MA. Basic kinematics of walking. Step length and step frequency. A Review. J Sport Med Phys Fit 1994; 34:109-34.

[25] Grieve DW, Gear RJ. The relationships between length of stride, step frequency, time of swing and speed of walking for children and adults. Ergonomics 1966;5:379-99.

[26] Rosenrot P, Wall JC, Charteris J. The relationship between velocity, stride time, support time and swing time during normal walking. J Hum Movement Stud 1980;6:323-35.

[27] Cavanagh PR, Kram R. Stride length in distance running: velocity, body dimensions and added mass effects. In: Cavanagh, editor. Biomechanics of distance running. Champaign, IL: Human Kinetics; 1990, p. 35-64.

[28] Dillman CJ. Kinematic analysis of running. Exercise Sport Sci Rev 1975;3:193-218.

[29] Lee CR, Farley CT. Determinants of the center of mass trajectory in human walking and running. J Exp Biol 1998;201:2935-44.

[30] Rosenbaum DA. Human motor control. Academic Press; 1991.

[31] Kelso JAS. Buchanan JJ, Murata T. Multifunctionality and switching in the coordination dynamics of reaching and grasping. Hum Movement Sci 1994;13:63-94.

[32] Verstappen M, Aerts P. Terrestrial locomotion in the black-billed magpie. Part I. Spatiotemporal gait characteristics. Motor Control 2000;4:150-64.

[33] Rubenson J, Heliams DB, Lloyd DG, Fournier PA. Gait selection in the ostrich: mechanical and metabolic characteristics of walking and running with and without an aerial phase. Proc R Soc 2004;271: 1091-9. 